\title{
A NeW Approach to the HISTOGram-BASEd SEgmentation OF Synthetic APERTURE RADAR IMAGES
}

Submitted: $6^{\text {th }}$ May 2020; accepted: $14^{\text {th }}$ March 2021

Barbara Siemiatkowska, Krzysztof Gromada

\section{DOI: 10.14313/JAMRIS/1-2021/5}

\begin{abstract}
:
Radar machine vision is an emerging research field in the mobile robotics. Because Synthetic Aperture Radars (SAR) are robust against weather and light condition, they provide more useful and reliable information than optical images. On the other hand, the data processing is more complicated and less researched than visible light images processing. The main goal of our research is to build simple and efficient method of SAR image analysis. In this article we describe our research related to SAR image segmentation and attempts to detect elements such as the buildings, roads and forest areas. Tests were carried out for the images made available by Leonardo Airborne \& Space System Company.
\end{abstract}

Keywords: SAR image, machine vision
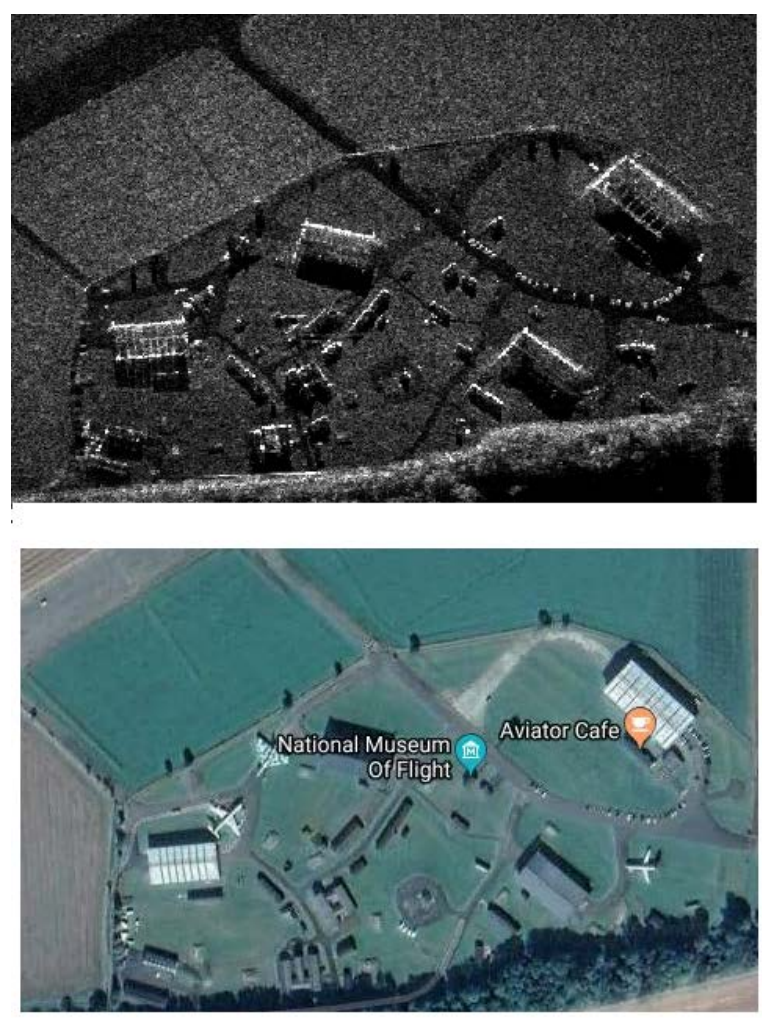

Fig. 1. Sample image: a - SAR image [source: Leonardo Airborne \& Space System], b - Google Map

\section{Introduction}

Nowadays, autonomous vehicles: cars, planes and drones are rapidly gaining popularity in both scientific research and practical applications, that involve dangerous rescue operations, airborne surveillance, product transport, military purposes but also autonomous cars. Such vehicles should be equipped with sensors that operate reliably regardless of weather conditions and time of a day. Modern autonomous vehicles, self-driving cars in particular, usually incorporate three kinds of sensors: camera, LiDAR and radar. LiDAR can accurately identify objects and their position, but it is expensive and susceptible to weather conditions.

Since 1978, Synthetic Aperture Radars (SAR) has been widely used for Earth remote sensing.

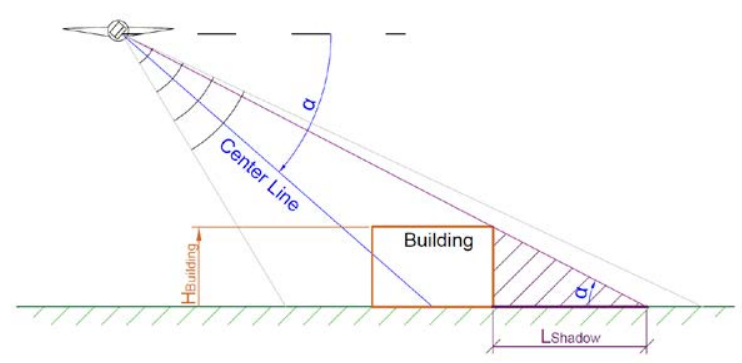

Fig. 2. Geometrical explanation of shadow appearance on SAR scans. Shadow area is marked with violet color, building - orange and ground - green

SARs use doppler effect to determine angle of received echo of electro-magnetic pulses (usually emitted by a built-in exciter, but can also use an external signal generator). Due to its operating principle SAR requires relative movement of a scanned object and a radar. Therefore SARs are usually mounted on moving platforms (typically a plane or satellite). The sensor provides high-resolution images which are independent of daylight and weather conditions [1].

The SAR system emits electromagnetic waves and receives altered echoes. The transmitted signal interacts with the Earth surface, and a portion of it is backscattered. The amplitude and phase of the echo depend on the properties (geometry, permittivity) of the surface and its position in regard to the sensor. The amplitude of SAR image represents a measure of the scene reflectivity.

The pulse frequency depends on the application. It ranges from single Megahertz for the spaceborne systems to hundreds of Gigahertz for the airborne approach. In case of the satellite systems, a single SAR scan provides information on an area of up to $400 \mathrm{~km}^{2}$. SAR has a wide range of applications: from planetary exploration, Earth monitoring, climate change research 
to agriculture, and military.

Radars are less expensive than LiDARs, but they are also less accurate. Many companies try to develop high-resolution radar and research is ongoing on the use of SAR radars in driver assistance systems [4]. SAR images are robust against different weather or daylight conditions [6], but they are usually affected by speckle noise [12], distortion effects like shadowing and high local contrasts in intensity. SAR images are challenging to interpret, their preprocessing and high level processing are very demanding tasks [11]. A SAR image is a valuable source of information about environment but it is very difficult to analyze.

Fig. 1 presents the SAR image of an area $7.33 \mathrm{~km}$ $\times 3.19 \mathrm{~km}$ and corresponding Google Map. Each pixel represents $1 \mathrm{~m} \times 1 \mathrm{~m}$ square. The image is very noisy, but the distinctive shadow effects can be seen.

Various image processing algorithms are used to support image analysis as well as significantly expand radar capabilities. The best-known is the Coherent Change Detection (CCD) algorithm [8], which allows us to detect minor differences in the height of the scanned area based on phase difference between two scans of the same area. CCD makes use of complex representation of SAR output (considering not only the amplitude of reflection, but also its phase). With current technology it gives resolution of up to 30 degrees of phase change (which for typical $3 \mathrm{GHz}$ frequency $10 \mathrm{~cm}$ wavelength - means around $30 / 360 \cdot 10 \mathrm{~cm}=$ $8 \mathrm{~mm}$ ) resolution.

However, when analyzing an individual image, it is very difficult to determine the type of surface of the observed objects (especially not man-made) or to define the boundaries between areas/objects. Thresholding method [9] is usually used for image segmentation. It is easy to implement, but provides low accuracy. Clustering methods [13] group pixels with similar properties and separate pixels which are different, thus method ensures higher accuracy but requires prior knowledge on expected parameters. Some authors propose to use deep learning neural networks [7] or SVM [14] classifiers for object recognition. Those methods require a big set of data, but without large public databases containing SAR images they are not available for civil or scientific use.

The main goal of our resarch is to build efficient method of SAR data analyzis. In this article, we describe our first steps towards new approach to SAR image segmentation and object classification. Our method combines adaptive thresholding and featurebased approach.

\section{Image Analysis}

\subsection{Image Histogram}

Unlike optical cameras, radar output image is not tightly related to its position during data gathering. Output scan is created by casting calculated amplitudes (and phases) of response on a plane (or sphere). Scan is virtually always a view from above of scanned area. This introduces an unintuitive effect of visible areas that were not scanned and thus are dark shadows in the output image.

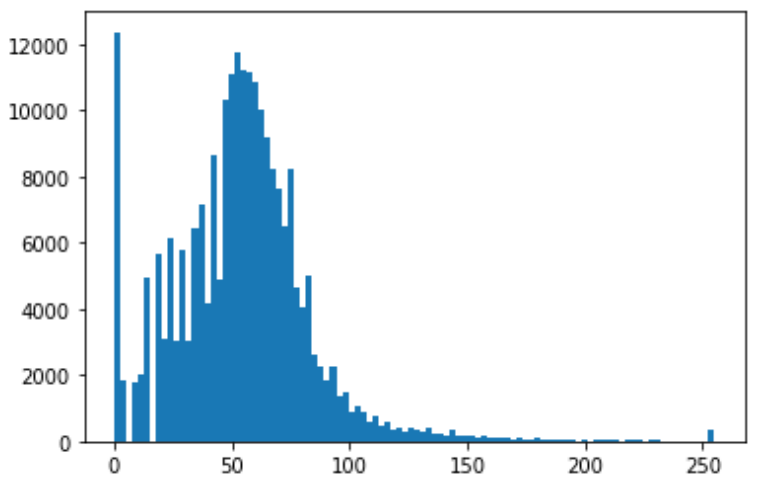

a

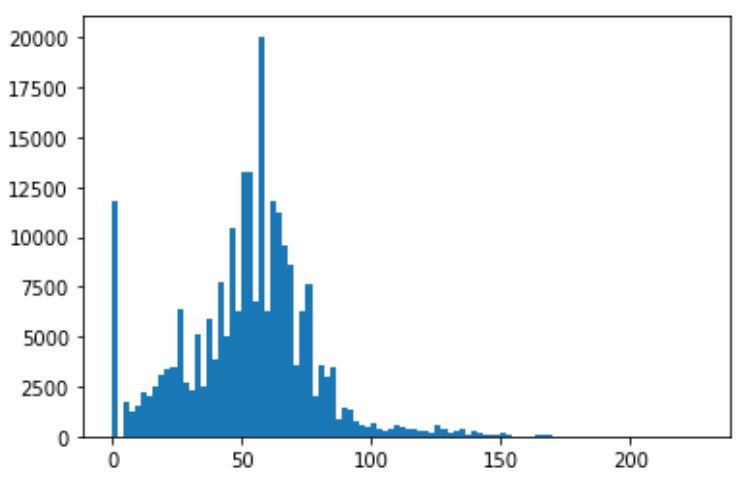

b

Fig. 3. Image histogram: a - raw image, b - filtered image

Fig. 2 presents the idea behind shadow creation. Radar needs to excite objects in area and receive their echo. Objects behind material that blocks electromagnetic waves do not backscatter a measurable amount of energy. For example, plastic does not block electromagnetic pulses. It is not detected by radar and casts no shadow on the image while we can notice the shadows near a building.

Knowing the scan angle $\alpha$ (often called look down angle) and measuring the shadow length $l_{\text {shadow }}$ (Fig. 2 ), building height $h_{\text {building }}$ can be determined using simple equation 1.

$$
H_{\text {Building }}=\tan (\alpha) \cdot L_{\text {Shadow }}
$$

Shadowing and high local contrasts in intensity effects mostly appear in SAR images of urban areas, and they are essential sources of information that are not available in optical images. For example, bright lines indicate the boundaries of buildings, and by analyzing shadows, it is possible to obtain a precise measurement of the height of an object.

Our goal is to perform image segmentation and to determine the area occupied by roads and buildings.

Usually the process of image analysis consists of the following modules [3]:

- noise reduction

- segmentation

- feature extraction

- final decision 


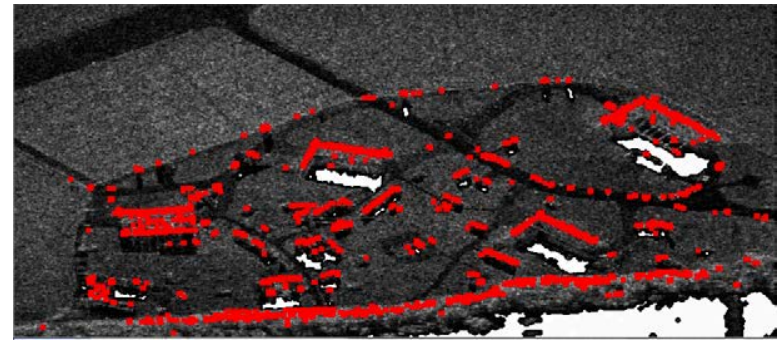

a

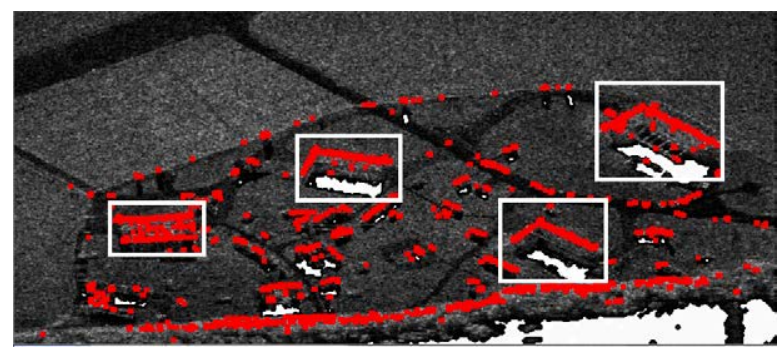

b

Fig. 4. a - a keypoints map, b - L-shape areas; red pixel represents a keypoint, white pixel - represents a shadow

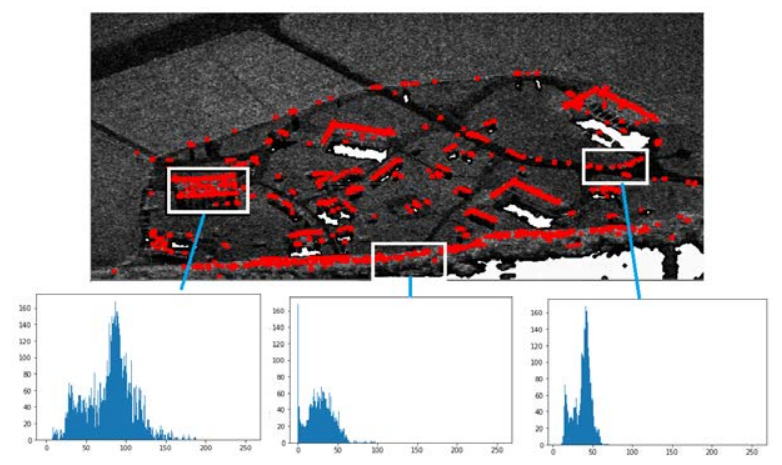

Fig. 5. Histograms of different areas

The method described in this article uses median filter for noise reduction [2]. This kind of filter preserves edges while removing noise. This feature is crucial for proper segmentation.

Low-lying areas and areas occupied by objects are analyzed differently. The first stage of our algorithm is to create a histogram. If we examine the histogram (Fig. 3), we can observe peaks. If a given pixel has a value of 0 , it is most likely a shadow fragment. If it is a light pixel, it is an element of a highly reflective object: a building, a car, etc. Bright and shadow (brightness $=0$ ) pixels will be key points and will be used in the process of segmentation and classification of objects. All other pixels will be treated as a part of the low-lying surface.

\subsection{Segmentation of Low-Lying Areas}

If we analyze the histogram, we can see that we cannot specify the threshold that will allow the segmentation of flat areas of different brightness. Therefore, at the first stage of the operation of our algorithm, median filtration with a $3 \mathrm{X} 3$ mask is per- formed several times. After completing this operation, we can notice the formation of peaks that will allow further segmentation. In our approach, multithresholding Otsu [10] algorithm is applied. In a classic method, the threshold is determined by minimizing intra-class intensity variance. The extension to multilevel thresholding was described in [5].

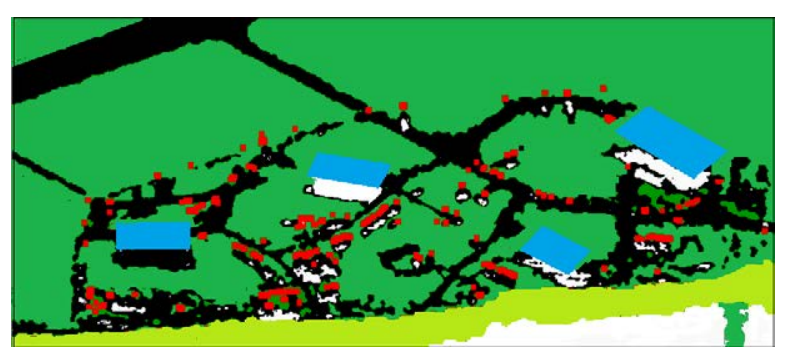

Fig. 6. The result of segmentation

\subsection{Segmentation of the Area Occupied by the Objects}

We assume that the bright pixels in the image correspond to the objects. These pixels are treated as key points, and their surroundings are analyzed. Figure $4 \mathrm{a}$ shows key points (red pixels). We can notice that if the bright pixels are arranged in the form of the letter $\mathrm{L}$, they represent a part of a building (Fig. 4b).

The L-shaped area can be easily detected using Hough transform. If the longest segments have been found, it is possible to build a parallelogram, which describes the visible part of the building. We can determine the height of the building based on the shape of the shadow, but this requires knowledge about the location of the aircraft.

Areas covered with trees can be distinguished from built-up areas by analyzing the histogram of a sharpened fragment of the image. For buildings, there are sharp edges, while for forests, the change in brightness is smooth. We can observe this difference by analyzing the histogram. Fig. 5 presents histograms of three different places: built-up areas (5a), cars (5c), forest (5b). In the last case, the value 0 is dominant in the histogram.

Figure 6 shows the final result of image segmentation. Two types of flat surface are detected (marked in black and dark green). Analyzing the shape of the areas, we can notice that the area marked in black is a fragment of the road. Light green pixels represent the forest. Detected buildings are represented as rectangles. Other key points (red - object and white - shadow) remained classified.

To carry out further classification, it is worth using contextual information. If the key point is surrounded by an area classified as a road, it can be considered a car, and if there is a shadow near it, it is most likely a tree.

\section{Conclusion}

This article describes our first research related to SAR image segmentation and attempts to detect elements such as buildings, roads, and forest areas. Be- 
cause of the lack of public databases containing SAR images, modern methods like deep learning methods or SVM cannot be applied. We have decided to use classic image processing methods. The algorithm combines histogram-based and simple rule-based systems. Tests carried out for SAR images made available by Leonardo Airborne \& Space System Company are promising. Further refinements are possible. Contextual information can be taken into account. The shape of a shadow can also be used to infer valuable information.

\section{ACKNOWLEDGEMENTS}

We thank Leonardo Airborne \& Space System Company for sharing SAR images and corresponding Google Map.

\section{AUTHORS}

Barbara Siemiatkowska* - Faculty of Mechatronics, Warsaw University of Technology, Warsaw, Poland, e-mail: siemiatkowska.barbara@pw.edu.pl.

Krzysztof Gromada* - PIT-RADWAR, Warsaw, Poland, e-mail: krzysztof.gromada@pitradwar.com.

*Corresponding author

\section{REFERENCES}

[1] A. Aguasca, R. Acevo-Herrera, A. Broquetas, J. J. Mallorqui, and X. Fabregas, "ARBRES: Light-Weight CW/FM SAR Sensors for Small UAVs", Sensors, vol. 13, no. 3, 2013, 3204-3216, $10.3390 / \mathrm{s} 130303204$.

[2] G. R. Arce, Nonlinear signal processing: a statistical approach, Wiley-Interscience: Hoboken, N.J, 2005.

[3] A. Fabijanska, M. Kuzanski, D. Sankowski, and L. Jackowska-Strumillo, "Application of image processing and analysis in selected industrial computer vision systems". In: 2008 International Conference on Perspective Technologies and Methods in MEMS Design, 2008, 27-31, 10.1109/MEMSTECH.2008.4558728.

[4] M. Farhadi, R. Feger, J. Fink, M. Gonser, J. Hasch, and A. Stelzer, "Adaption of Fast Factorized BackProjection to Automotive SAR Applications". In: 2019 16th European Radar Conference (EuRAD), 2019.

[5] D.-Y. Huang and C.-H. Wang, "Optimal multilevel thresholding using a two-stage Otsu optimization approach", Pattern Recognition Letters, vol. 30, no. 3, 2009, 275-284, 10.1016/j.patrec.2008.10.003.

[6] Y. Huang and Y.-C. Huang, "Segmenting SAR Satellite Images With the Multilayer Level Set Approach", IEEE Journal of Selected Topics in Applied Earth Observations and Remote Sensing, vol. 4, no. 3, 2011, 632-642, 10.1109/JSTARS.2011.2158390.
[7] F. Lattari, B. Gonzalez Leon, F. Asaro, A. Rucci, C. Prati, and M. Matteucci, "Deep Learning for SAR Image Despeckling", Remote Sensing, vol. 11, no. 13, 2019, 1532, 10.3390/rs11131532.

[8] A. V. Monti-Guarnieri, M. A. Brovelli, M. Manzoni, M. Mariotti d'Alessandro, M. E. Molinari, and D. Oxoli, "Coherent Change Detection for Multipass SAR", IEEE Transactions on Geoscience and Remote Sensing, vol. 56, no. 11, 2018, 68116822, 10.1109/TGRS.2018.2843560.

[9] G. Moser and S. Serpico, "Generalized minimumerror thresholding for unsupervised change detection from SAR amplitude imagery", IEEE Transactions on Geoscience and Remote Sensing, vol. 44, no. 10, 2006, 2972-2982, 10.1109/TGRS.2006.876288.

[10] M. Sezgin and B. Sankur, "Survey over image thresholding techniques and quantitative performance evaluation", Journal of Electronic Imaging, vol. 13, no. 1, 2004, 146-165, $10.1117 / 1.1631315$.

[11] X. Xue, H. Wang, F. Xiang, and J. Wang, "A new method of SAR Image Segmentation Based on FCM and wavelet transform". In: 2012 5th International Congress on Image and Signal Processing, 2012, 621-624, 10.1109/CISP.2012.6469844.

[12] H. Yu, X. Zhang, S. Wang, and B. Hou, "ContextBased Hierarchical Unequal Merging for SAR Image Segmentation", IEEE Transactions on Geoscience and Remote Sensing, vol. 51, no. 2, 2013, 995-1009, 10.1109/TGRS.2012.2203604.

[13] Y. Zhong, A. Ma, and L. Zhang, "An Adaptive Memetic Fuzzy Clustering Algorithm With Spatial Information for Remote Sensing Imagery", IEEE Journal of Selected Topics in Applied Earth Observations and Remote Sensing, vol. 7, no. 4, 2014, 1235-1248, 10.1109/JSTARS.2014.2303634.

[14] P. Zhou, G. Guo, and F. Xiong, "Research on modified SVM for classification of SAR images". In: Proceedings of the 2017 International Conference on Frontiers of Manufacturing Science and Measuring Technology, 2017. 